\title{
Nanocarrier Applied in The Distribution of Drugs in the Assistance for the Cancer Treatment
}

\author{
María Isabel Araya González ${ }^{1}$, Lizeth Alejandra Castro Quirós ${ }^{1}$, Manuel Jesús Guevara Elizondo ${ }^{1}$, \\ Joel Josue Ramírez Alfaro ${ }^{1}$ and Jose Vega Baudrit ${ }^{2 *}$ \\ ${ }^{1}$ Escuela de Química, Universidad Nacional, Costa Rica \\ ${ }^{2}$ Laboratorio Nacional de Nanotecnología LANOTEC-CeNAT-CONARE, Costa Rica
}

*Corresponding author: Jose Vega Baudrit, Laboratorio Nacional de Nanotecnología LANOTEC-CeNAT-CONARE, San José, Costa Rica.

To Cite This Article: Jose Vega Baudrit. Nanocarrier Applied in The Distribution of Drugs in the Assistance for the Cancer Treatment. Am J Biomed Sci \& Res. 2019 - 5(6). AJBSR.MS.ID.000970. DOI: 10.34297/AJBSR.2019.05.000970

Received: 啙 October 10, 2019; Published: 盋 October 21, 2019

\begin{abstract}
Cancer is the disease that takes more human lives worldwide, currently the treatments available for this disease are very aggressive, among which chemotherapy stands out. As an alternative to these treatments, studies have been carried out for the synthesis of nanocarriers which have as an advantage over conventional treatments, an increase in the solubility of the drug, allow a more specific delivery of this and a lower renal clearance; Among the wide range of materials with which they can be manufactured include dendrines, liposomes and micelles. These manage to be assimilated by the organism through different biological routes distributed either by sustained liberation or response to stimuli. Although the nanocarriers present different challenges, the avenues in the area have allowed them to overcome them in order to be a treatment with smaller cytotoxicity and better efficiency at the preclinical level than conventional treatments.
\end{abstract}

Keywords: Nanocarriers; Cancer; Drug delivery; Opsonization; Biodistribution

\section{Introduction}

GLOBOCAN 2018 estimated 18.1 million cases per year worldwide, which is expected to increase to 29.5 million cases of cancer per year by 2040 [1]. Overall, cancer is an uncontrolled proliferation of cells that generate tumor masses, with the exception of hematologic cancers that spread through the lymphatic, blood and bone marrow systems. Tumors grow thanks to angiogenesis, which is the new vascularization created to supply oxygen to cancer cells. At the same time, cancer cells possess replicative immortality and resistance to death, so that over time it spreads to other parts of the body and eventually causes death [2]. The origin of cancer is due to a mutation of the proto-oncogenes that encode the proteins involved in cell proliferation and differentiation, as well as the genes that encode the proteins that inhibit cell growth and stimulate apoptosis [3]. The most common treatments for non-metastatic cancers are surgery and radiation therapy, but when the cancer has spread throughout the body, these treatments are ineffective [4].

The main option currently available for treating metastatic cancers is chemotherapy, as it is able to reach all organs of the body through the bloodstream. Chemotherapy is based on cytotoxic compounds that inhibit the rapid proliferation of cancer cells, but unfortunately also affects normal cells with rapid proliferation rates such as hair follicles, bone marrow and gastrointestinal tract cells. Consequently, there is the manifestation of side effects observed in cancer treatment using chemotherapy [4]. In addition to the strong side effects of conventional chemotherapy, there are other factors that disadvantage the method, such as low oral bioavailability, high toxicity, low water solubility, low therapeutic indices, inconsistent circulation, non-specific biological distribution [5]. Given the negative characteristics of conventional chemotherapy, the doors have been opened to research and implementation of targeted therapies in the late 1990s [4].

Nanotechnology has provided promising advantages for targeted chemotherapy delivery systems. Due to the unique size, high surface area and ease of manipulation of the surface area to implement selectivity, nanotransporters are considered the ideal platform for the transport of chemotherapeutic drugs [6]. In addition to transporting chemotherapeutic agents to tumors, avoiding normal tissues and reducing toxicity in the rest of the body, they also protect cytotoxic drugs from degradation, increase 
half-life, payload, solubility of cytotoxic agents and reduce renal clearance [2]. These nanocarriers are nanoscale colloidal systems capable of transporting anti-cancer agents, such as low molecular weight drugs or macromolecules such as genes or proteins, so that these anti-cancer agents avoid normal tissues and accumulate only in tumors. This results in a cytotoxic concentration several times higher in tumors, with reduced toxicity for the rest of the body compared to free medicines [7].

As a result, several types of nanotransport systems have been developed for the administration of pharmaceuticals from a large number of active constituents. Nanotransport systems mostly include polymer nanoparticles, nanoliposomes, dendrimers, niosomes, polymer micelles, solid lipid nanoparticles. For targeted drug delivery, nanotransporters are modified with specific ligands on their surface to improve selective cell uptake, as well as to reduce non-specific biodistribution [8]. As mentioned, nanocarriers comprise mainly polymeric therapeutic products: polymer-protein and polymer-pharmaceutical conjugates, in which the drug is covalently joined or conjugated with a polymeric structure, and drug nanocarriers, where the drug is physically trapped within molecular assemblies with different structures made of different polymeric materials.

Polymer nanoparticles are the most effective carriers for the delivery of drugs aimed at controlled and prolonged cancer. These biodegradable colloidal systems include spherical polymer particles of nanometric size, where cytotoxic drugs can be encapsulated or physically trapped within a matrix polymer substance or trapped in a cavity surrounded by a polymer membrane, and it is also possible to conjugate the anti-cancer drug to the surface or nucleus of the particles [9]. In the case of insoluble drugs, it is possible to produce a hydrophobic interaction between the drug and the nucleus of the particle, increasing its solubility. When the drug conjugates with the particle, the properties of the binders play a crucial role in the pharmacological properties of the complex, they can make them stable in the bloodstream at $\mathrm{pH} 7$ and decompose at $\mathrm{pH} 5.5$, the typical $\mathrm{pH}$ in tumors, or be stable in the blood, but be divided by lysosomal enzymes in tumors [10].

In addition to their protective properties, these carriers also offer: multiple supply of synergistic drugs, reduced toxicity with limited interaction with healthy cells, prolonged circulation times, increased uptake by cancer cells, features that they share with other nanotransporters, but also show: better stability, more homogeneous size distribution, more controllable physicochemical properties, higher drug payload and more controlled drug release through diffusion through the polymer matrix or through particle erosion and degradation, compared to other colloidal systems [11].

\section{Nanotransporters}

Nanotechnology offers incredible potential in the pharmaceutical and medical fields in the diagnosis and treatment of cancer, producing multifunctional treatments that package functions such as tissue implantation, tissue regeneration, etc., in a fraction of the size of a cell [12]. Despite the rise of nanotechnologies, the definition of these remains a controversial issue, as there is no universally accepted classification. For the purpose of its study, nanomedicine has been operationally defined as the use of nanoscale elements or nanostructured materials in medicine, which are designed to have unique medical effects thanks to their structure. Their main characteristic is their size, up to $300 \mathrm{~nm}$, which allows them to take advantage of two phenomena that occur on the nano scale, transitions of properties and physicochemical interactions [12].

The concept that nanomedicines aim to improve the therapeutic index of cancer drugs by modifying their pharmacokinetics and tissue distribution to improve delivery to the site of action is well known and has also been clinically demonstrated [13]. This is thanks to the fact that they allow both the transport of low molecular weight drugs and genetic agents such as oligonucleotides [14]. This coincides with the definition of the "ideal transporter" for cancer treatment, which must have the ability to simultaneously transport both chemotherapeutic drugs and genetic agents to the cancer cells, releasing the payloads in a controlled manner and exact dose, thus achieving a maximum effect of combined therapy for the treatment of drug-resistant tumors [2,15].

Nanomaterials have a number of advantages as drug carriers. Nanocarriers can:

i. Increase water solubility and protect drugs dissolved in the bloodstream, improving the pharmacokinetic and pharmacological properties of drugs.

ii. Aim at the delivery of drugs in a specific way of tissue or cell, thus limiting the accumulation of drugs in the kidneys, liver, spleen and other undirected organs and improving therapeutic efficacy.

iii. Provide a combination of imaging and therapeutic agents for real-time monitoring of therapeutic efficacy [14].

Another strong point of the use of nanomedicines is the "clearance" or renal clearance which is a pharmacological parameter of the speed with which waste substances are removed from the body, taking into account both metabolic processes through the liver and excretion through the kidneys. The reduction of clearance goes hand in hand with an increase in the half-life of nanomedicine and thus an increase in efficiency and bioavailability [16]. Although there is no official classification as mentioned above, nanoparticles can be roughly divided into inorganic, lipidic and polymeric. Inorganic nanoparticles are a generic term that includes several nanoparticles such as gold, Quantum dots, metal oxides, metal nanostructures, superparamagnetic oxides and carbon nanotubes [17]. 
Organic nanoparticles, also known as solid lipids, have the advantages and avoid the disadvantages of other colloidal transporters such as emulsions and liposomes; they generally have conformations such as nanocapsules and dendrines. Finally, polymer nanoparticles include natural, biocompatible and biodegradable polymers. This means that they do not alert the immune system and break down into harmless metabolic compounds [18]. Most of these have the adequate size $(10-100 \mathrm{~nm})$ to penetrate the capillaries and enter the tissues, this also allows them to have greater specificity [17]. One of the main advantages of using nanotechnology in medicine is its specificity, since thanks to the effect known as MPR (improvement, permeability and retention), specificity is provided from size and geometry, which allows targeting a specific tissue. This is usually known as passive specificity, where molecular targets are used, overexpressed or expressed exclusively on the surface of tumor cells [2], while, if some additional effect is used, such as alteration of specific cell signaling events by means of monoclonal antibodies or small molecule inhibitors to improve drug specificity, it is known as active specificity [12].

Thanks to this high specificity, nanomedicines accumulate in high concentrations, but only in the affected site, which allows a decrease in the systematic toxicity of drugs, increasing the therapeutic potential of drugs and increasing the retention time in the body [18], even in sites complicated to focus such as the brain [17]. This is achieved because nanoparticles, which can be adapted to have various biological properties and can be used in a variety of environments thanks to their size, allowvolumes to be distributed up to three times larger than by traditional means and can additionally be used in complicated media, such as intracranially, as they provide a safer and more effective means for the delivery of medicine such as chemotherapy [14]. In 2012 it was demonstrated by treating an intracranial tumor that drug-loaded polymer nanoparticles (PLGA nanoparticles loaded with camptotecin) allow localized delivery to the tumor site [19]. Although studies are underway with innovative strategies to improve active specificity, there is much debate as to whether this is really necessary to achieve effective accumulation in the affected tissue. The most commonly used targeting techniques are antibody fragments, antigen ligands and variable chain fragments [20]. While nanomaterials have many associated benefits and have become important, it is important to take into account and assess the inherent limitations of the materials included, such as stability, specificity, efficiency and toxicity [21].

\section{Physical Characteristics}

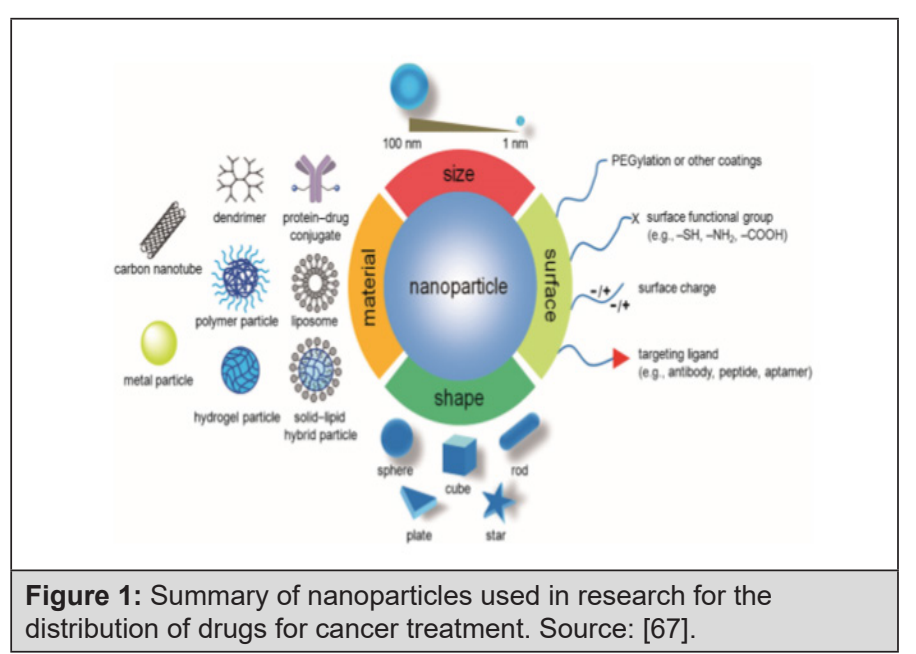

A selective delivery system based on polymer nanoparticles is characterized by biodegradability, biocompatibility, non-toxicity, prolonged circulation and a broad spectrum of payload of a therapeutic agent. Recent progress in the field of targeted drug delivery system is based on rational polymer design (composition, solubility, crystallinity, molecular weight, skeletal stability, hydrophobicity and polydispersion) adapted to a particular charge with respect to molecular weight and drug load [22]. Figure 1 summarizes the most significant variables of the polymeric nanomaterials used to design the nanotransporters. This figure details different morphologies, range of sizes, types of surfaces and materials from which they are formed.

\section{Types of Nanotransporters}

Table 1: Different types of nanotransporters, with their polymeric composition, active compound and therapeutic use.

\begin{tabular}{|c|c|c|c|c|}
\hline Nanotransporters & Material/ Polymer & $\begin{array}{l}\text { Encapsulated Active } \\
\text { Compound }\end{array}$ & Therapeutic use / benefits & References \\
\hline \multirow{6}{*}{ Nanoparticle } & $\begin{array}{c}\text { Poly ( } \varepsilon \text {-caprolactone) / eudragit RS } \\
\text { 100; Poly (lactida-co-glicolida) / } \\
\text { eudragit RS } 100\end{array}$ & Insulin & Diabetes mellitus & {$[88,105]$} \\
\hline & Alginate/ dextran sulfate & Insulin & Diabetes mellitus & {$[103,104]$} \\
\hline & \multirow{4}{*}{ PLGA } & Paclitaxel & Improves antitumor efficacy & [94] \\
\hline & & Dexamethasone & $\begin{array}{l}\text { Improves transplacental } \\
\text { permeability }\end{array}$ & [85] \\
\hline & & Estradiol & Brain distribution & [98] \\
\hline & & Doxorubicin & $\begin{array}{l}\text { Improves oral biodistribution, } \\
\text { reduces cardiotoxicity }\end{array}$ & [93] \\
\hline Micelles & Chitosan N-octil-N-arginine & Insulin & Diabetes mellitus & [74] \\
\hline
\end{tabular}




\begin{tabular}{|c|c|c|c|c|}
\hline & $\begin{array}{l}\mathrm{N} \text {-deoxicholic-N, O-hydroxiethyl } \\
\text { chitosan (DHC) acid }\end{array}$ & Paclitaxel & Improve biodistribution & {$[53]$} \\
\hline \multirow[b]{2}{*}{ Liposomes } & chitosan & Alendronate & Osteoporosis & {$[90]$} \\
\hline & Polyacrilic acid & Paclitaxel & $\begin{array}{l}\text { Improves oral bioavailability, } \\
\text { reduces tumor growth }\end{array}$ & [92] \\
\hline Niosomes & Span 60 , cholesterol & Cefdinir & Sustained in vitro release up to $12 \mathrm{~h}$ & [86] \\
\hline \multirow[t]{2}{*}{ Dendrines } & $\begin{array}{l}\text { Fatty acid, phospholipid coated } \\
\text { polyamidoamine }\end{array}$ & 5-fluorouracil & Improves oral bioavailability & Cheng et al. (2007) \\
\hline & G3 polyamidoamine & Propranolol & Improves oral bioavailability & [100] \\
\hline $\begin{array}{l}\text { Nanoparticles } \\
\text { lipidic solid }\end{array}$ & $\begin{array}{l}\text { Egg lecithin, pepsin, diethylene } \\
\text { glycol monoethyl ether }\end{array}$ & Triptolide & Reduces gastric irritation & {$[74]$} \\
\hline \multirow{2}{*}{ Nanocapsules } & $\begin{array}{l}\beta \text {-lactoglubulin, low methoxy pectin, } \\
\text { bovine milk }\end{array}$ & Oxali-palladium & Colon cancer & [89] \\
\hline & Chitosan & Campotecine & $\begin{array}{l}\text { Improves oral bioavailability, } \\
\text { reduces dose frequency }\end{array}$ & [102] \\
\hline
\end{tabular}

Nanotransport systems generally have different morphologies such as: nanoparticles, micelles, hydrogels, nano sponges, liposomes, nano adhesives, among others [8]. As an example, Table 1 presents examples of different types of nanotransporters applied to a plethora of active compounds to achieve different diseases [6]. A timely choice of morphology can be an improvement in different properties of the nanotransporters such as bioavailability and a better location of the specific site of interest [23]. This bibliographic review focuses on the following morphologies: dendrines, liposomes and micelles.

\section{Dendrins}

The term dendrines is derived from the Greek dendro meaning "tree" and groupers meaning "part". Defined as spherical macromolecules, monodisperses, synthetic, highly branched, with three-dimensional nanometric configurations [20]. How a dendrin manages to interact with the drug, as well as with genetic molecules are based on: simple encapsulations, electrostatic interactions and covalent conjugations, due to empty internal cavities [24]. Dendrines have a large number of advantages such as: greater drug release capacity in the tumour, better permeability of the drug, as well as an increase in the half-life, solubility and stability of the drug. Dendrines are nanostructures obtained from macromolecules such as polyamidoamine (PAMAM) and polypropyleneimine; and they are highly branched with an internal nucleus. They are unique because they have series of branches, multivalent, well-defined molecular weight and globular structure with controlled surface functionality. The presence of a multivalent nature allows the union of several drug molecules, addressing groups and solubilization groups on their surfaces in a well-defined manner.

The latter explains the versatility of including both hydrophobic and hydrophilic groups in its structure. [25,26]. This allows a greater effectiveness of the drug in the treatment, since solubility plays an important role in bioavailability $[20,27,28]$. Devarakonda and company compared the solubility potential of dendrines and PANAM's dextrin cycle in increasing niclosamide solubility and observed a greater capacity in improving dendrin solubility
$[29,30]$. Another advantage in the application of dendrins is the incorporation of degradable bonds that can be used in the release of the drug. Chang [31] proposes the release of the drug by means of a conjugated system of folic acid with polyethylglycol dendrimers (PEG) (PAMAM) with doxorubicin (DOX) and super-paramagnetic iron oxide. In this system the release of the drug responds to the pH of the medium, which allowed the controlled release of DOX in tampons with $\mathrm{pH}$ similar to endosomes and lysosomes of tumor cells to be treated [31].

In spite of having a great number of advantages when applying systems with dendrimers, there is the existence of statics that recognize the toxicity problems for this type of morphology due to its size, as well as the presence of functional groups with positive charges especially with amino groups. Due to this characteristic size and functional group, a non-specific interaction with mitochondria, enzymes and cell membranes results [23]. Different authors qualify its use as an alternative for cancer treatment in a pragmatic way $[32,15]$. The decrease in dendrin toxicity can be improved by conjugating a single pass, such as Cong [32] bioconjugated glycyrrhetinic acid with propyleneimine dendrimers by obtaining high quality nanotransporters through in vivo assays [32](Figure 2).

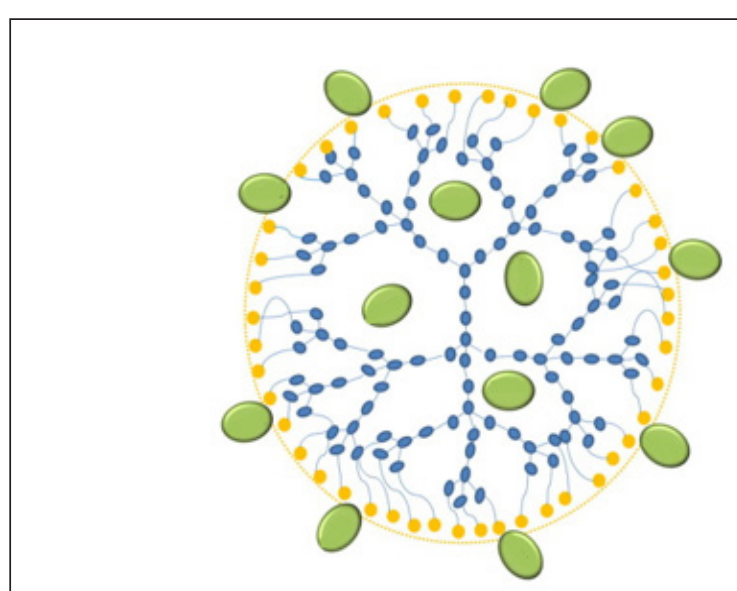

Figure 2: Diagram of drug load in a dendrin. Source [23]. 


\section{Liposomes}

Liposomes consist of an aqueous nucleus surrounded by one or more layers of phospholipids and cholesterol which can form a lipid layer. These can be classified according to the number of layers per particle and its average size: it is classified as a small mono-laminar vesicle (SUV's) if its average size is in the range of $15-100 \mathrm{~nm}$ and as a large mono-laminar vesicle (LUV's) when its size range is around $100 \mathrm{~nm}$ to $1000 \mathrm{~nm}$ and finally as multilaminar vesicles with an interval of $1 \mu \mathrm{m}$ to $5 \mu \mathrm{m}$ [34]. Liposomes have a series of advantages over other types of morphology such as protection against drug degradation in the medium, thus allowing a slower and more controlled release, also having a lipid bilayer will give a decrease in the leakage of the drug to the medium [35-37]. This morphology is considered to be the most effective transporter for the distribution of chemotherapy drugs [15].

The mechanism of diffusion of drugs from the liposome to the tumour will depend on the polymer used, since it may be by means of a destabilisation of the lipid bilayer or by a fusion of the liposome with the endosome of the cell [17]. Despite the advantages mentioned above, the disadvantage is the vulnerability of the liposomes through phagocytosis by macrophages derived from the reticular system (RES) $[15,38]$, giving it poor physical stability compared to other types of transporters [39]. In order to improve localization selectivity, multicomponent liposome formulations have been developed [40], Chen and collaborators in 2015 applied a liposome modified with glycyrrhetinic acid in order to load oxaliplatin for liver-directed bio-distribution studies, where the results suggest that the modified liposome has an excellent tissuespecific targeting [41]. Likewise, the use of cationic liposomes has been used in order to improve the transport of chemotherapies, with 1,2-dioleoyl-3-trimethylammonium-propane (DOTAP) being the most common [42]. These charged liposomes are synthesized by electrostatic interactions by lipidic cations and negatively charged phosphates.

Liposomes have been used in the formulation of different transporters for cancer treatment using drugs such as doxorubicin, daunorubicin and citrabine, for ovarian cancer, however, these can not only be applied as a transporter in chemotherapy treatment, but also in gene therapy treatments, with the formation of the RNA/ liposome complex [43]. Feng and collaborators in 2014 synthesized a liposome/RNA complex modified with vapreotide, this is a somatostatin analogue that has a high affinity with somatostatin receptors (SSTR), which are expressed in excess in many tumor cells. The nanoparticle nucleus corresponds to a negatively charged ternary complex composed of RNAsi, chondroitin sulfate and protamine, and could be coated with a layer of cationic lipids. As a result, the mixed liposome had a significantly stronger drug distribution in tumor tissues through receptor-directed administration [44](Figure 3).

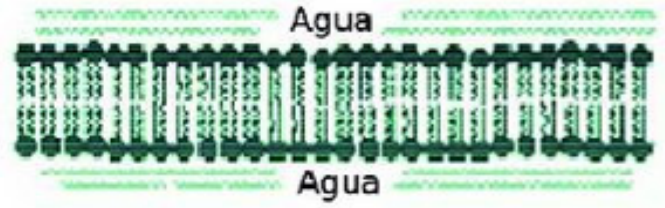

(a)

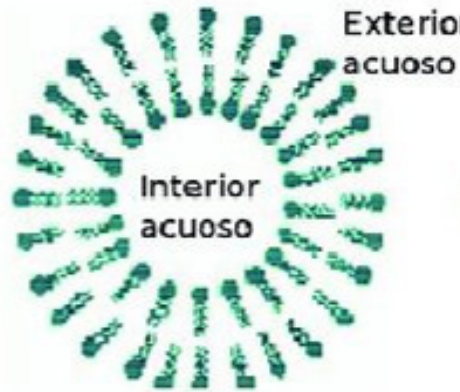

(b)

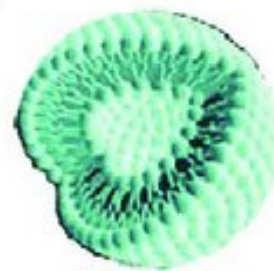

(c)

Figure 3: Scheme of a liposome: (a) view of its lipidic bi-layer; (b) view of a cross section; (c) view of a three-dimensional cut. Source: [97].

\section{Micelles}

Micelles consist of an amphiphilic block of copolymers, these pose both hydrophobic and hydrophilic groups, these first are oriented towards the interior of the mycel, while the hydrophilic form a kind of external armour. The hydrophobic group acts as a reserve for drugs that are poorly soluble in water [15]. The drug is encapsulated during the emulsification process and is released during degradation in the group of cells of interest [45], micelles are mostly applied in passive treatments exploiting the permeability and retention effect (EPR) of tumor tissues [46,47]. These nanocarriers can be formulated from various biocompatible polymers such as chitosan, PEG, methacrylate acid (MAA), methyl methacrylate (MMA), PMAA, poly (amido amine), (PAMAM) poly (l-aspartic acid) (PAsp), N-isopropylacrylamide (NIPAM), dimethylacrylamide (DMAA), 10-undecenoic acid (UA), poly (10-undecenoic acid) (PUA), 2-hydroxyethyl methacrylate (HEMA), ethylacrylate (EA), ethyl methacrylate (EMA), butyl methacrylate (BMA), N- (2-hydroxypropyl) methacrylamide (HPMA) octadecyl acrylate (ODA), N-vinyl-2-pyrrolidone (PV), poly (N, N-Methyl dimethylaminoacetacrylate) (DMAEMA), polyglutamic acid (PGA), poly (d, l-lactide) (PLA), poly ( $\varepsilon$-caprolactone) (PCL) and poly-d, l-lactide-co-glycolide (PLGA) [38].

These types of nanotransporters have a number of advantages such as their biocompatibility, as well as good physiological 
stability due to a low critical micellar concentration [39], allowing the formation of micelle at low concentrations of surfactant [38]. Also, due to the size of the hydrophilic cortex there is the formation of a smooth surface, which reduces interactions with proteins, allowing a longer time of circulation in the blood [48] Among the disadvantages that the micelles have, they can be dissociated to the point of dissolving, this together with the low load of the drug results in the release of the drug before reaching the tumor area [23]. In order to give solution to this problem, intercrossing is proposed, however, a very stable mycel was obtained as a result, causing a slow release of the drug. Therefore, it is proposed to apply response stimuli to the polymeric mycelae such as temperature and pH [49-53](Figure 4).

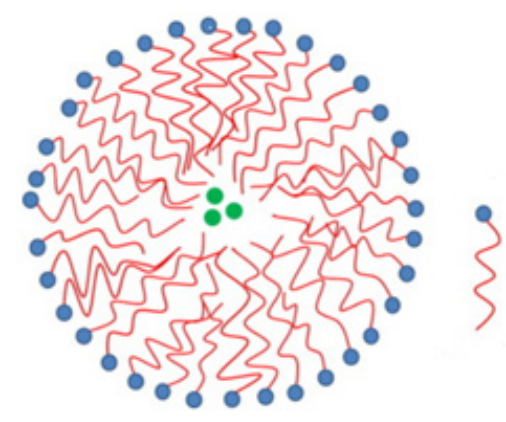

Figure 4: Diagram of a micelle. Source [23]

\section{Biological Assimilation, Opsonization and Biodistribution of Drugs by Means of Nanoparticles}

The greatest challenge of using a new drug to combat cancer is its specificity in terms of its distribution route and dose. Current chemotherapy strategies are far from ideal for cancer treatment, as they are generally associated with serious side effects, low bioavailability and resistance to multiple drugs. As an alternative to achieve better performance and efficiency in the administration of a drug, nanoparticles are used as means of facilitating transport and carriers of the substances that develop the activity [54]. For a drug delivery device to achieve these desired benefits, it must be present in the bloodstream long enough to reach or recognize its therapeutic site of action. However, the opsonization or elimination of drug carriers (nanoparticles) from the body by the mononuclear phagocyte system (MSF), also known as the reticuloendothelial system (RRS), is a major obstacle to achieving these goals [55].

\section{Opsonization}

Opsonization is the process by which a foreign organism or particle is covered with opsonine proteins, which makes it more visible to phagocytic cells. After opsonization, phagocytosis may occur, which is the destruction or removal of foreign materials from the bloodstream. Together, these two processes form the primary clearance mechanism for undesirable components larger than the renal threshold limit of the blood. In the case of polymer nanoparticles, which normally cannot be destroyed by phagocytes, sequestration in the organs of SFM usually occurs. If the polymeric nanoparticle is not biodegradable, the accumulation of particles in these organs, usually the liver and spleen, can produce toxicity and other negative side effects [55]. Generally speaking, opsonsins are any component of the blood serum that aids in the phagocytic recognition process, but complement proteins such as $\mathrm{C} 3, \mathrm{C} 4$ and C5 and immunoglobulins are usually the most common. Several methods of camouflage or masking of nanoparticles have been developed that allow them to temporarily avoid recognition by SFM and increase their half-life in blood circulation [56]. Opsonization usually takes place in the blood circulation and may take from a few seconds to several days to complete [55].

SFM macrophages have the ability to remove unprotected nanoparticles from the bloodstream within seconds of intravenous administration, rendering them ineffective as site-specific drug delivery devices [57]. Temporary control of a drug's release may also help decrease unwanted side effects that occur due to natural circadian fluctuations in chemical levels throughout the body [58], in addition to more targeted action without loss of drugs by not achieving the desired effect. To achieve this distribution control, the use of nanoparticles has many distinct advantages, including adjustable size, modifiable surface, high load capacity, improved drug bioavailability, controlled drug release, and precise tumor targeting; thus, nanoparticle drug delivery systems are highly effective in maximizing therapeutic efficacy and minimizing toxicity [59-62].

As mentioned above, immunoglobulins such as C3, C4, and C5 are common opsonins, as well as other blood serum proteins such as laminin, fibronectin, C-reactive protein, type I collagen, and many others. Opsonins, which are present throughout the blood, are believed to come into contact with injected polymer nanoparticles, typically by random Brownian motion. However, once close enough to the surface of a particle, any intermolecular force such as van der Walls, electrostatic, ionic, hydrophobic/hydrophobic, and others may be involved in binding opsons to the surface of the nanoparticle [55]. A stable relationship must be fostered between the characteristics of the drug, together with its nanocarriers, in order to resist the onslaughts of the opsonsins and overcome other mechanisms of protection of the organism such as cell membranes. Some hydrophobic drugs may not be soluble enough to pass through the aqueous environment (e.g., the body and tissue fluids in vivo) surrounding a cell and then penetrate the cell membrane to eventually reach intracellular targets. On the other hand, its strong tendency to aggregate in intravenous administration can lead to complications such as embolisms and local toxicity [63].

Once opsonization has occurred, the phagocyte binds to the nanoparticle through opsons attached to the surface. To accomplish this purpose, phagocytic cell surfaces contain specialized receptors that interact with the modified conformation of these opsonins, 
alerting them to the presence of foreign material. A second method of phagocyte binding is non-specific adherence of phagocytes to blood serum proteins adsorbed to the surface, which may also result in stimulation of phagocytosis. Complement activation may also occur. Regardless of the activation pathway, the final result is the binding and phagocytosis of the foreign particle by mononuclear phagocytes [55]. The third and final step in the purification process is the ingestion of foreign materials by the phagocytes. This step in the process usually involves endocytosis of the particle or foreign material by a phagocyte. After particle endocytosis, the phagocytes will begin secreting enzymes and other chemical factors reactive to oxidation, such as superoxides, oxyhalide molecules, nitric oxide, and hydrogen peroxide, to break down the phagocyte material.

Unfortunately, most non-biodegradable polymer nanoparticles cannot be significantly degraded by this process and, depending on their relative size and molecular weight, will be either removed by the renal system or sequestered and stored in one of the SFM organs. To improve drug delivery performance and avoid significant opsonization and phagocyte elimination losses, emphasis should be placed on placing drug molecules in or on the surface of a nanoparticle carrier that allows controlled release, offering multiple benefits compared to conventional dosage forms based on free drugs. For example, it can improve the temporal and spatial presentations of a drug in the body, protect the drug from physiological degradation or elimination, reduce toxicity to healthy tissues and organs, and increase patient compliance and comfort [64].

\section{Distribution mechanisms}

Polymer nanoparticles have been used as the preferred vehicle for drug delivery at the nanoscale, especially for their excellent efficacy in endocytosis, passive orientation of the tumor, high encapsulation efficiency and delivery of a wide range of therapeutic agents [65]. However, these polymeric substances with controllable surface load and monodispersed size distribution are synthetically challenging [66]. Great strides have been made in the design and development of nanoparticle-based systems for controlled release, and their modes of operation can be classified into two main categories: sustained release and stimulus sensitive or also known as intelligent response substances [67].

Figure 5 shows the different routes by which the polymeric nanomaterial together with the drug enters the target tissue, or the drug manages to interact with the target tissue. Among the systems that can be developed are endocytosis, by means of proteins and other groups that allow the intrusion of the desired substance in the damaged cells; diffusion in the case of small drugs and phagocytosis and pinocytosis depending on the size of the particles.

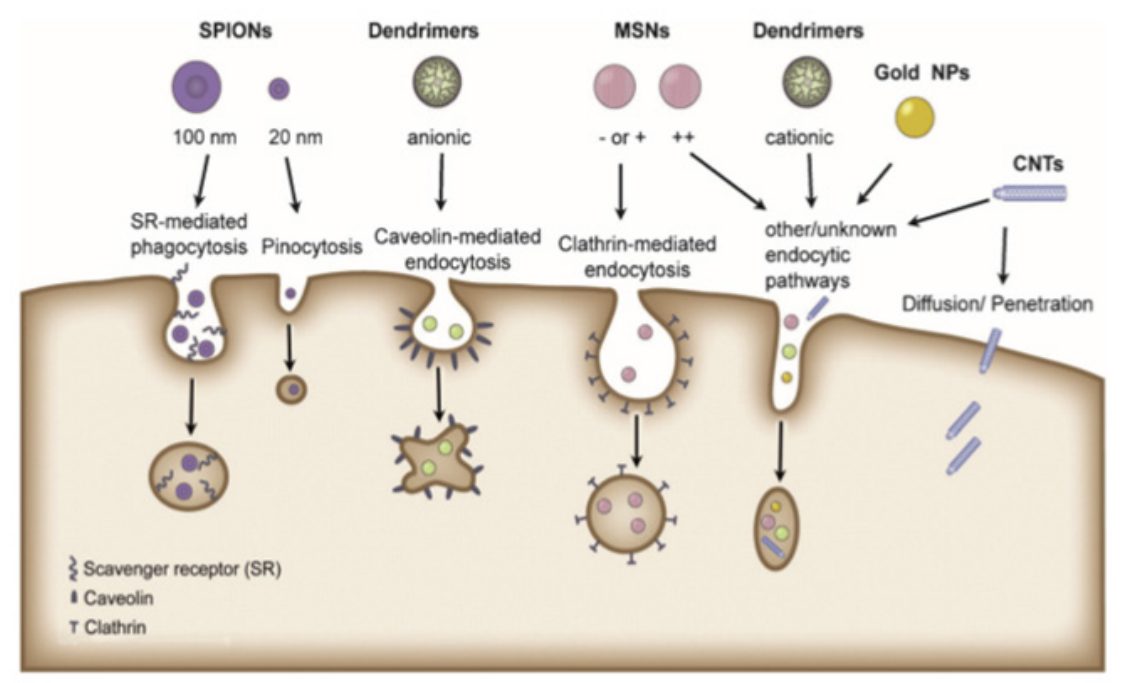

Figure 5: Routes of entry of different types of nanoparticles into target cells. T. G. Iversen, T. Skotland, K. Sandvig, Nano Today 2011, 6, 176-185.

Sustained release: Sustained release aims to deliver a medication at a predetermined rate

over a prolonged period of time. This mode of release is critical for drugs that are quickly metabolized and removed from the body after administration. Sustained release allows the drug concentration to be maintained at a constant level in the plasma or target tissue by matching the drug release rate with the drug elimination rate. In the case of cancer therapy, keeping the concentration of a drug within the therapeutic window is beneficial to the patient [67]. Once dissolved in the aqueous body fluid, most drugs can be transported freely with the fluid to quickly reach target receptors. One approach to achieving sustained release is to prevent drug molecules from entering the aqueous environment for a controllable period of time; this prevention can be accomplished by controlling the diffusion of drug molecules through an insoluble polymer layer or matrix, or by simply controlling the rate of transporter degradation [68]. Release mechanisms and corresponding mathematical models have been extensively studied and reviewed [69]. 
a)
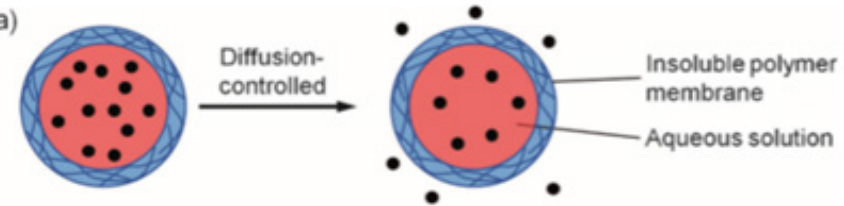

b)
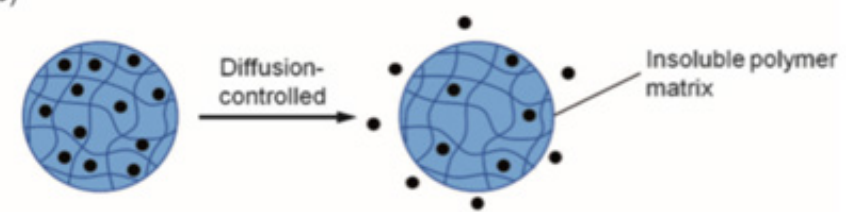

c)
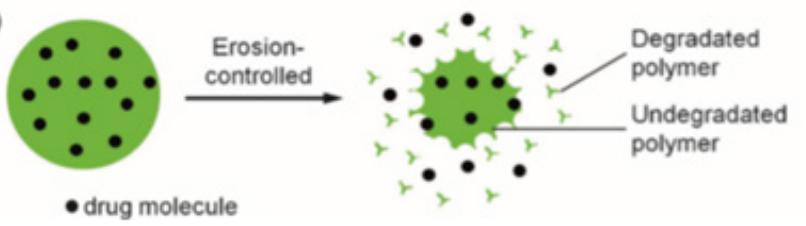

Figure 6: Main mechanisms for achieving sustained drug release: $a, b)$ diffusion through an insoluble polymer layer or matrix, c) erosion of a polymer matrix. Source [68]

Figure 6 presents examples of sustained release by different types of nanoparticle morphologies containing the drug to be distributed. It is possible to appreciate the different alternatives used in which a controlled concentration of the drug in the medium is regulated when the polymer is released, either by its diffusion through the membrane or matrix formed by the nanotransporter, or by the degradation over time experienced by the vehicle when exposed to the medium. As observed in figure 6, in diffusion controlled release, preloaded drug molecules within a nanoparticle have restricted access to the aqueous environment through a barrier provided by an insoluble material, which corresponds to a nanoscale organic polymer in most cases. In general, controlled release by diffusion can be performed using a reservoir-based system such as liposomes or micelles, or matrices that behave like scaffolds [70].

By name, a reservoir-based system consists of a central conformation containing the drug and a membrane surrounding the reservoir. The concept of this release system can be easily extended to nanometric carriers by switching to colloidal hollow particles [15]. Typically, drug molecules initially charged into the reservoir can only diffuse across the membrane. On the other hand, drug nanocarriers made of erodible or degradable polymers have attracted much attention because they do not require recovery or further manipulation after the drug has been completely released [41]. The pattern of drug release can be controlled by adapting the erosion kinetics of nanoparticles through careful selection of polymers and encapsulation techniques [71] and other surrounding tissues are not put at risk because degradation of the polymer allows it to be discarded like any other harmless low molecular weight substance, such as renal elimination [55].

\section{Stimulus release (intelligent response): everal environmentally sensitive polymeric}

nanoparticles have been actively developed that dissolve, swell or collapse in response to an internal stimulus, for example: $\mathrm{pH}$, glucose, redox potential and lysosomal enzymes; or external stimuli such as temperature, magnetic field, ultrasound and light, to achieve improved drug release at the target site (spatial control) and at the appropriate time (time control) [72,73]. For example, taking advantage of slightly acidic environments in cancerous tissues ( $\mathrm{pH}$ 6.5-7.2), endosomes ( $\mathrm{pH}$ 5.0-6.5) and lysosomes ( $\mathrm{pH}$ 4.5-5.0) compared to a physiological $\mathrm{pH}$ of 7.4 in blood and normal tissues. These nanoparticles that respond to internal stimuli have the advantages of self-controlled drug release and easy application in clinical settings [74].

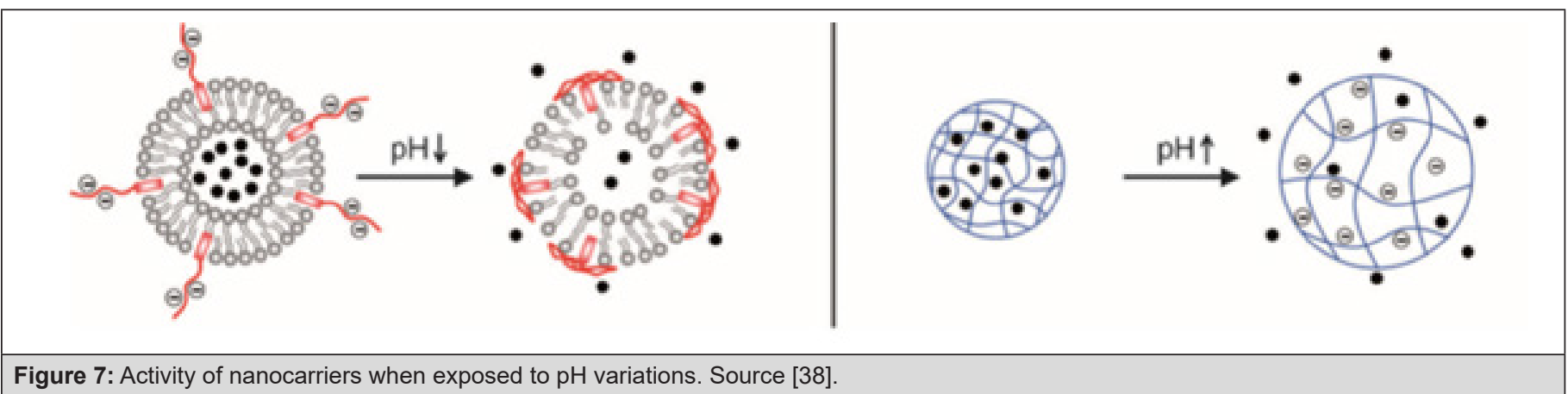


In the case of nanoparticles that take advantage of variations in the $\mathrm{pH}$ of the medium, the aim is to use polymers with functional groups that can alter the density of charges in response to variations in $\mathrm{pH}$. Notable examples include poly (acrylamide) (PAAm), poly (acrylic acid) (PAA), poly (methacrylic acid) (PMAA), poly (methyl acrylate) (PMA), poly (diethylaminoethyl methacrylate) (PDEAEMA) and poly (dimethylaminoethyl methacrylate) (PDMAEMA). With the use of these polymers, the structure and hydrophobicity of nanoparticle carriers may change as a result of protonation or deprotonation. $\mathrm{pH}$-sensitive nanoparticles have been designed and developed to release drugs at the tumor site or endo / lysosomal compartments (Figure 7).

Enzymatic activity can break certain bonds, causing disassembly or destruction of structures [75]. These enzymatic reactions can be used to trigger the release of a drug. More significantly, altered expression of a specific enzyme associated with a pathological condition can be used to achieve enzyme-mediated drug release only at the desired biological target [76]. An enzyme-sensitive release system includes a structural scaffold that is susceptible to degradation by a specific bond between the drug and the carrier as a product of an enzymatic reaction [77]. Hydrolases are the most commonly used enzymes for such an application, as they can break covalent bonds or modify certain chemical groups by altering the balance between electrostatic, hydrophobic and van der Waals forces, $p$ - p interactions, or hydrogen bonds [75,78]. Specific cases occur in tumor tissues, which are highly hypoxic with levels of glutathione (GSH) at least four times higher than normal tissues [79], a variable with great potential to combat cancer cells.

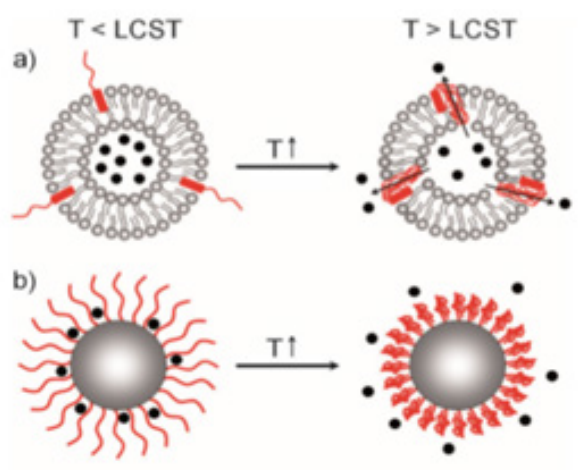

Figure 8: Influence of temperature on thermosensitive nanocarriers. Source: [101].

There are other external factors that can induce a response in the controlled release of drugs such as magnetic field, ultrasound, light and temperature [79]. The release of drugs using temperature is among the most researched strategies for the intelligent administration of drugs because it can take advantage of the increase in local temperature caused by the pathological condition (for example, tumor, inflammation or infection). Thermosensitive release is based on a sudden change in the physical properties of a temperature-sensitive material. Such an acute response can trigger the release of the drug if the local temperature around the carrier changes. The temperature range, within which the drug delivery system is activated, should be maintained between 37 and $42^{\circ} \mathrm{C}$, as temperatures beyond this range will cause denaturation of proteins or disruption of function [80] (Figure 8).

The use of light is presented as an important external factor, using photosensitivity for the introduction of nanoparticles through functional groups that can change their conformations or other properties after irradiation by light with an appropriate wavelength [74]. Notable examples include azobenzene, pyrene, nitrobenzene, cinnamoyl and spirobenzopyrene. The reversible molecular change of the azobenzene group (and its derivatives) from strangulation irradiated by UV light (300-380nm) and from cis to trans by visible light allows photoregulated control of drug release [81]. Another alternative is the use of magnetic nanoparticles (MNP), which have received great interest due to their various potential biomedical applications, including the administration of targeted drugs, magnetic thermotherapy (hyperthermia), magnetic resonance imaging (MRI) and the separation of proteins and cells [82]. In particular, super-paramagnetic magnetite (Fe304) has attracted the most attention due to its unique magnetic properties and biocompatibility [83] (Figure 9).

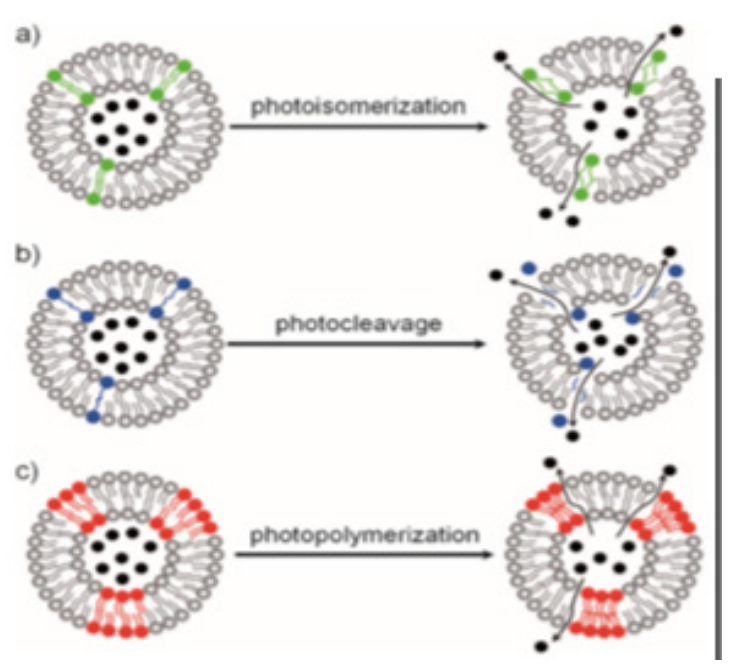

Figure 9: Photosensitive nanotransport activity when exposed to a light source. Source [95].

Challenges in the Area of Nanoparticles as Nanotransporters of Drugs

The advance of nanomedicine therapies to the market is often slow. This may be because their clinical effectiveness is not sufficient to justify accelerated development, or because technical or cost challenges in expansion and manufacturing may delay (or require more) investment. Much less research effort has been devoted to the challenge of understanding the correlations between patient biology and nanomedicine behavior [13]. In particular, combined therapeutic regimens for different types of cancer remain a challenge due to the various mechanisms of cancer development. Combination therapy with nanoparticle drug carriers therefore 
warrants further study at the preclinical and clinical levels. There are other challenges for modified and functionalized nanomaterials with well-established formulations, including improved localization, biodistribution, biocompatibility and efficacy of in vivo nanodrates systems, to meet the requirements of precision cancer diagnosis and therapy [14].

Nanotechnology can help improve the distribution and selection of antitumor drugs. The distribution of cancer drugs is defined by their physicochemical properties and is limited by drug penetration into tumor tissue. Not all nanotransporters penetrate intrinsically into tumor tissue which remains a current challenge [84]. The main administration challenges for these agents include significant off-target accumulation, crossing the cell membrane, achieving synergistic drug proportions in the target and a narrow therapeutic window. Turning these next generation therapies "impossible to deliver" into viable cancer treatments has become one of the major future directions for nanomedicines [13]. A better understanding of the biology of the tumor's microenvironment is also needed. The implications of genetic and molecular markers should be encouraged by inventing active tumor detection strategies using nanoparticles to predict disease progression, development and clinical outcomes [22,85-106].

\section{Conclusion}

The research carried out in the field of nanotechnology has allowed an improvement in the systems of nanotransporters with different morphologies, controlling their different disadvantages, allowing the criterion for choosing the type of morphology to be the need for treatment. Many authors point out that the biospecificity that nanotransporters can develop contributes to reducing cytotoxicity in significant levels, thus avoiding the affectation or death of healthy tissue. Efficiencies achieved at the preclinical level show very favorable results given the low renal clearance of drugs compared to nonspecific drugs. The great variety of compounds applicable to this field of nanomaterials has an important relevance in terms of the discovery of new nanotransporters, biological disposition and assimilation in the target cells, due to the versatility that these materials have to behave differently in organisms depending on the site to be submitted to treatment.

\section{References}

1. Bray F, Ferlay J, Soerjomataram I, Siegel RL, Torre LA, et al. (2018) Global cancer statistics 2018: GLOBOCAN estimates of incidence and mortality worldwide for 36 cancers in 185 countries. CA Cancer J Clin 68(6): 394424.

2. Pérez E, Fernández A (2015) Advanced targeted therapies in cancer: Drug nanocarriers, the future of chemotherapy. Eur J Pharm Biopharm 93: 52-79.

3. Hanahan D, Weinberg R (2011) Hallmarks of cancer: the next generation. Cell 144(5): 646-674.

4. Chabner B, Roberts T (2005) Timeline: chemotherapy and the war on cancer. Nat Rev Cancer 5(1): 65-72.

5. Soni G, Yadav K (2015) Applications of nanoparticles in treatment and diagnosis of leukemia Mater Sci Eng C Mater Biol Appln 47: 156-164.
6. Mukherjee B, Satapathy B, Bhattacharya S, Chakraborty R, Mishra $\mathrm{V}$ (2017) Pharmacokinetic and pharmacodynamic modulations of therapeutically active constituents from orally administered nanocarriers along with a glimpse of their advantages and limitations. Nano- and microscale drug delivery systems. Elsevier Inc Pp. 357-375.

7. Danhier F, Feron O, Préat V (2010) To exploit the tumor microenvironment: passive and active tumor targeting of nanocarriers for anti-cancer drug delivery. J Control Release 148(2): 135-146.

8. Mukherjee B, Satapathy B, Mondal L, Dey N, Maji R (2013) Potentials and challenges of active targeting at the tumor cells by engineered polymeric NPs. Curr Pharm Biotechnol 14(15): 1250-1263.

9. Hillaireau H, Couvreur P (2009) Nanocarriers' entry into the cell: relevance to drug delivery. Cell Mol Life Sci 66(17): 2873-2896.

10. Ulbrich K, Šubr V (2004) Polymeric anticancer drugs with pH-controlled activation Adv. Drug Deliv Rev 56(7): 1023-1050.

11. Hu C, Aryal S, Zhang L (2010) Nanoparticle-assisted combination therapies for effective cancer treatment Ther Deliv 1(2): 323-334.

12. Etheridge M, Campbell S, Erdman A, Haynes C, Wolf S, et al. (2013) The big picture on nanomedicine: The state of investigational and approved nanomedicine products. Nanomedicine 9(1): 1-14.

13. Hare JI, Lammers T, Ashford MB, Puri S, Storm G, et al. (2017) Challenges and strategies in anti-cancer nanomedicine development: An industry perspective. Adv Drug Deliv Rev 108: 25-38.

14. Li Y, Xiao K, Luo J, Xiao W, Lee JS, et al. (2011) Well-defined, reversible disulfide cross-linked micelles for on-demand paclitaxel delivery. Biomaterials 32(27): 6633-6645.

15. Li Z, Tan S, Li S, Shen Q Wang K (2017) Cancer drug delivery in the nano era: An overview and perspectives. Oncology Reports 38(2): 611-624.

16. Bregoli L, Movia D, Gavigan Imedio JD, Lysaght J, Reynolds J, et al. (2016) Nanomedicine applied to translational oncology: A future perspective on cancer treatment. Nanomedicine 12(1): 81-103.

17. Nazir S, Hussain T, Ayub A, Rashid U, MacRobert AJ (2014) Nanomaterials in combating cancer: Therapeutic applications and developments. Nanomedicine: Nanomedicine 10(1): 19-34.

18. Lenjisa J, Woldu M, Satessa G (2014) New hope for eradication of HIV from the body: The role of polymeric nanomedicines in HIV/AIDS pharmacotherapy. J Nanobiotechnology 12(1): 9.

19. Patel T, Zhou J, Piepmeier JM, Saltzman WM (2012) Polymeric nanoparticles for drug delivery to the central nervous system. Adv Drug Deliv Rev 64(7): 701-705.

20. Jain N, Tekade R (2013) Dendrimers for enhanced drug solubilization. Drug delivery strategies for poorly Water-Soluble drugs Oxford. UK: John Wiley \& Sons Ltd Pp. 373-409.

21. Cho H, Lee Y (2014) Nano-sized drug delivery systems for lymphatic delivery. J Nanosci Nanotechnol 14(1): 868-880.

22. Masood F (2016) Polymeric nanoparticles for targeted drug delivery system for cancer therapy. Mater Sci Eng C Mater Biol Appl 60: 569-578.

23. Venditti I (2017) Morphologies and functionalities of polymeric nanocarriers as chemical tools for drug delivery: A review. Journal of King Saud University - Science 31(3): 398-411.

24. Cheng Y, Xu T, Xu Z, Ma M (2008) Dendrimers as drug carriers: Applications in different routes of drug administration. J Pharm Sci 97(1): 123-143

25. Imae T, Cheng Y (2012) Physicochemical properties of dendrimers and dendrimer complexes Dendrimer-Based Drug Delivery Systems. John Wiley \& Sons Inc Hoboken NJ Pp. 55-92.

26. Wolinsky JS, Grindstaff MW (2008) Therapeutic and diagnostic applications of dendrimers for cancer treatment. Adv Drug Delivery Rev 60(9): 1037-1055. 
27. Boghra R, Kothawade P, Belgamwar V (2011) Solubility, dissolution rate and bioavailability enhancement of irbesartan by solid dispersion technique. Chem Pharm Bull (Tokyo) 59(4): 438-441.

28. Guo F, Zhong H, He J (2011) Self-micro emulsifying drug delivery system for improved oral bioavailability of dipyridamole: Preparation and evaluation, Arch Pharm Res 34(7): 1113-1123.

29. Devarakonda B, Hill RA, Liebenberg W, Britts M, DeVilliers MM (2005) Comparison of aqueous solubilization of practically insoluble niclosamide by polyamidoamine (PAMAM) dendrimers and cyclodextrins. Int J Pharm 304(1-2): 193-209.

30. Devarakonda B, Hill RA, DeVilliers MM (2004) The effect of PAMAM dendrimer generation size and surface functional group on the aqueous solubility of nifedipine. Int J Pharm 284(1-2): 133-140.

31. Chang Y, N Liu, L Chen, X Meng, Y Liu, et al. (2012) Synthesis and characterization of DOX-conjugated dendrimer-modified magnetic iron oxide conjugates for magnetic resonance imaging, targeting, and drug delivery. J Mater Chem 22: 9594-9601.

32. Gandhi N, Tekade R, Chougule M (2014) Nanocarrier mediated delivery of siRNA/miRNA in combination with chemotherapeutic agents for cancer therapy: Current progress and advances. J Control Release 194: 238-256.

33. Cong Y, Shi B, Lu Y, Wen S, Chung R, et al. (2016) One-step conjugation of glycyrrhetinic acid to cationic polymers for high-performance gene delivery to cultured liver cell. Sci Rep 6: 21891.

34. Fahr A, Liu X (2007) Drug delivery strategies for poorly water-soluble drugs. Expert Opin Drug Deliv 4(4): 403-416.

35. Scherphof G, Roerdink F, Waite M, Parks J (1978) Disintegration of phosphatidylcholine liposomes in plasma as a result of interaction with high-density lipoproteins. Biochim Biophys Acta 542(2): 296-307.

36. Allen T, Cleland L (1980) Serum-induced leakage of liposome contents. Biochim Biophys Acta 597(2): 418-426.

37. Senior J, Gregoriadis G (1982) Is half-life of circulating liposomes determined by changes in their permeability?. FEBS Lett 145(1): 109114.

38. Felber A, Dufresne M, Leroux J (2012) pH-sensitive vesicles, polymeric micelles, and nanospheres prepared with polycarboxylates. Drug Delivery Rev 64(11): 979-992.

39. Tsouris V, Joo MK, Kim SH, Kwon IC, Won YY (2014) Nano carriers that enable co-delivery of chemotherapy and RNAi agents for treatment of drug-resistant cancers. Biotechnol Adv 32(5): 1037-1050.

40. Irvine D (2011) Drug delivery: One nanoparticle, one kill. Nat Mater 10: 342-343.

41. Ma Y, Zheng Y, Zeng X, Jiang L, Chen H, et al. (2011) Novel docetaxelloaded nanoparticles based on PCL-Tween 80 copolymer for cancer treatment. Int J Nanomedicine 6: 2679-2688.

42. Skandrani N, Barras A, Legrand D, Gharbi T, Boulahdour H, et al. (2014) Lipid nanocapsules functionalized with polyethyleneimine for plasmid DNA and drug co-delivery and cell imaging. Nanoscale 6(13): 73797390 .

43. Lee J, Lee M (2012) Liposome mediated cancer gene therapy: Clinical trials and their lessons to stem cell therapy. Bull Korean Chem Soc 33(2): 433-442.

44. Feng Q Yu MZ, Wang JC, Hou WJ, Gao LY, et al. (2014) Synergistic inhibition of breast cancer by co-delivery of VEGF siRNA and paclitaxel via vapreotide-modified core-shell nanoparticles. Biomaterials. 35(18): 5028-5038.

45. Miyata K, Christie R, Kataoka K (2011) Polymeric micelles for nano-scale drug delivery. React Funct Polym 71(3): 227-234.

46. Bae Y, Kataoka K (2009) Intelligent polymeric micelles from functional poly (ethylene glycol)-poly (amino acid) block copolymers. Adv Drug Deliv Rev 61(10): 768-784.
47. Tyrrell Z, Shen Y, Radosz M (2010) Fabrication of micellar nanoparticles for drug delivery through the self-assembly of block copolymers. Prog Polym Sci 35(9): 1128-1143.

48. Jiang X, Sha X, Xin H, Chen L, Gao X, et al. (2011) Self-aggregated pegylated poly (trimethylene carbonate) nanoparticles decorated with $\mathrm{c}(\mathrm{RGDyK})$ peptide for targeted paclitaxel delivery to integrin-rich tumors. Biomaterials 32(35): 9457-9469.

49. Fujimori J, Yoshihashi Y, Yonemochi E, Terada K (2005) Application of Eudragit RS to thermo-sensitive drug delivery systems: II. Effect of temperature on drug permeability through membrane consisting of Eudragit RS/PEG 400 blend polymers. Control Release 102(1): 49-45.

50. Chan Y, Wong T, Byrne F, Kavallaris M, Bulmus V (2008) Acid-labile core cross-linked micelles for $\mathrm{pH}$-triggered release of antitumor drugs. Biomacromolecules 9(7): 1826-1836.

51. Dai J, Lin S, Cheng D, Zou Z, Shuai X (2011) Interlayer-crosslinked micelle with partially hydrated core showing reduction and $\mathrm{pH}$ dual sensitivity for pinpointed intracellular drug release. Angew Chem Int Ed Engl 50(40): 9404-9408

52. Song N, Liu W, Tu Q, Liu R, Zhang Y, et al. (2011) Preparation and in vitro properties of redox-responsive polymeric nanoparticles for paclitaxel delivery. Colloids Surf B Biointerfaces 87(2): 454-563.

53. Li H, Huo M, Zhou J, Dai Y, Deng Y, et al. (2010) Enhanced oral absorption of paclitaxel in N-deoxycholic acid-N, O-hydroxyethyl chitosan micellar system. J Pharm Sci 99(11): 4543-4553.

54. Mishra D, Hubenak J, Mathur A (2013) Nanoparticle systems as tools to improve drug delivery and therapeutic efficacy. J Biomed Mater Res A 101(12): 3646-3660.

55. Owens D, Peppas N (2006) Opsonization, biodistribution, and pharmacokinetics of polymeric nanoparticles. Int J Pharm 307(1): 93102.

56. Kaul G, Amiji M (2002) Long-circulation poly (ethylene glycol) modified gelatin nanoparticles for intracellular delivery. Pharm Res 19(7): 10611067.

57. Gref R, Minamitake Y, Peracchia M, Trubetskoy V, Torchilin V, et al. (1994) Biodegradable long-circulating polymeric nanospheres. Science 263(5153): 1600-1603.

58. Hermida R, Fernández J, Ayala D, Mojon A, Alonso L, et al. (2001) Circadian rhythm of double (rate-pressure) product in healthy normotensive young subjects Chronobiol Int 18(3): 475-489.

59. Rink J, Plebanek M, Tripathy S, Thaxton C (2013) Update on current and potential nanoparticle cancer therapies. Curr Opin Oncol 25(6): 646651.

60. Parveen S, Misra R, Sahoo S (2012) Nanoparticles: a boon to drug delivery, therapeutics, diagnostics and imaging. Nanomedicine $8(2)$ : 147-166.

61. Zhong YN, Goltsche K, Cheng L, Xie F, Meng FH, et al. (2016) Hyaluronic acid-shelled acid-activatable paclitaxel prodrug micelles effectively target and treat CD44 overexpressing human breast tumor xenografts in vivo. Biomaterials 84: 250-261.

62. Zhu DW, Tao W, Zhang HL, Liu G, Wang T, et al. (2016) Docetaxel (DTX)loaded polydopamine-modified TPGS-PLA nanoparticles as a targeted drug delivery system for the treatment of liver cancer. Acta Biomater 30 : 144-154.

63. Owen S, Doak A, Wassam P, Shoichet M, Shoichet B (2012) Colloidal Aggregation Affects the Efficacy of Anticancer Drugs in Cell Culture. ACS Chem Biol 7(8): 1429-1435.

64. Wang Y, Shim M, Sung H, Xia Y (2014) Stimuli-Responsive Materials for Controlled Release of Theranostic Agents. Advanced Functional Materials 24(27): 4206-4220.

65. Faraji A, Wipf P (2009) Nanoparticles in celular drug delivery. Bioorg Med Chem 17(8): 2950-2962. 
66. Lu F, Wu S, Hung Y, Mou C (2009) Size effect on cell uptake in wellsuspended, uniform mesoporous silica nanoparticles. Small 5(12): 1408-1413.

67. Sun T, Zhang Y, Pang B, Hyun D, Yang M, et al. (2014) Engineered Nanoparticles for Drug Delivery in Cancer Therapy. Angew Chem Int Ed Engl 53(46): 12320-12364.

68. Robitzki A, Kurz R (2010) Biosensing and drug delivery at the microscale: novel devices for controlled and responsive drug delivery. Handb Exp Pharmacol 197: 87-112.

69. Siepmann J, Siepmann F (2008) Mathematical modeling of drug delivery. Int J Pharm 364(2): 328-343.

70. Pundir S, Badola A, Sharma D (2013) Sustained release matrix technology and recent advance in matrix drug delivery system: a review. Int J Drug Res Technol 3: 12-20.

71. Zilberman M, Grinber O, Biomater J (2008) HRP-loaded bioresorbable microspheres: Effect of copolymer composition and molecular weight on microstructure and release profile. Appl 22(5): 391-407.

72. Ganta S, Devalapally H, Shahiwala A, Amiji M (2008) A review of stimuliresponsive nanocarriers for drug and gene delivery. J Control Release 126(3): 187-204

73. Rapoport N, Zhong Z, Feijen J (2009) Physical stimuli-responsive polymersomes for programmed drug delivery Biomacromolecules 10(2): 197-209.

74. Zhang Y, Yin Q, Yin L, Ma L, Tang L, et al. (2013) Chain-Shattering Polymeric Therapeutics with On-Demand Drug-Release Capability. Angew Chem Int Ed Engl 52(25): 6563-6567.

75. Lorenzo C, Concheiro A (2013) From Drug Dosage Forms to Intelligent Drug-delivery Systems: a Change of Paradigm. Smart Materials for Drug Delivery Pp. 1-32.

76. Xia X, Yang M, Oetjen L, Zhang Y, Li Q, et al. (2011) An enzyme-sensitive probe for photoacoustic imaging and fluorescence detection of protease activity. Nanoscale 3: 950-953.

77. Cutler J, Auyeung E, Mirkin C (2012) Spherical Nucleic Acids J Am Chem Soc 134: 1376-1391.

78. Law B, Tung C (2009) Proteolysis: A Biological Process Adapted in Drug Delivery, Therapy, and Imaging. Bioconjugate Chem 20(9): 1683-1695.

79. Cheng R, Meng F, Deng C, Klok H, Zhong Z (2013) Dual and multi-stimuli responsive polymeric nanoparticles for programmed site-specific drug delivery. Biomaterials 34(14): 3647-3657.

80. Xiong M, Bao Y, Yang X, Wang Y, Sun B, et al. (2012) Lipase-Sensitive Polymeric Triple-Layered Nanogel for "On-Demand” Drug Delivery. J Am Chem Soc 134(9): 4355-4362.

81. Sortino S (2012) Photoactivated nanomaterials for biomedical release applications. J Mater Chem 22(2): 301-318.

82. Shubayev V, Pisanicli T, Jin S (2009) Magnetic nanoparticles for theragnostics. Adv Drug Deliv Rev 61(6): 467-477.

83. Hu F, Wei I, Zhou Z, Ran Y, Li Z, et al. (2006) Preparation of biocompatible magnetite nanocrystals for in vivo magnetic resonance detection of cancer. Adv Mater 18(19): 2553-2556.

84. Wicki A, Witzigmann D, Balasubramanian V, Huwyler J (2015) Nanomedicine in cancer therapy: Challenges, opportunities, and clinical applications. J Control Release 200: 138-157.

85. Ali H, Kalashnikova I, White MA, Sherman M, Rytting E (2013) Preparation, characterization, and transport of dexamethasone-loaded polymeric nanoparticles across a human placental in vitro model. Int J Pharm 454(1): 149-157.

86. Bansal S, Aggarwal G, Chandel P, Harikumar SL (2013) Design and development of cefdinir niosomes for oral delivery. J Pharm Bioallied Sci 5(4): 318-325.
87. Chen J, Jiang H, Wu Y, Li Y, Gao Y (2015) A novel glycyrrhetinic acidmodified oxaliplatin liposome for liver-targeting and in vitro/vivo evaluation. Drug Des Devel Ther 9: 2265-2275.

88. Damage C, Maincent P, Ubrich N (2007) Oral delivery of insulin associated to polymeric nanoparticles in diabetic rats. J Control Release 117(2): 163-170

89. Ghalandari B, Divsalar A, Saboury A, Parivar K (2014) The new insight into oral drug delivery system based on metal drugs in colon cancer therapy through $\beta$-lactoglobulin/oxali-palladium nanocapsules. J Photochem Photobiol B 140: 255-265.

90. Han H, Shin H, Ha D (2012) Improved oral bioavailability of alendronate via the mucoadhesive liposomal delivery system. Eur J Pharm Sci 46(5): 500-507.

91. Iversen T, Skotland K, Sandvig (2011) Endocytosis and intracellular transport of nanoparticles: Present knowledge and need for future studies. Nano Today 6(2): 176-185.

92. Jain S, Kumar D, Swarnakar N, Thanki K (2012) Polyelectrolyte stabilized multilayered liposomes for oral delivery of paclitaxel. Biomaterials 33(28): 6758-6768.

93. Kalaria D, Sharma G, Beniwal V, Kumar M (2009) Design of biodegradable nanoparticles for oral delivery of doxorubicin: in vivo pharmacokinetics and toxicity studies in rats. Pharm Res 26(3): 492-501.

94. Kumari A, Yadav S, Yadav S (2010) Biodegradable polymeric nanoparticles based drug delivery systems. Colloids Surf B Biointerfaces 75(1): 1-18

95. Leung S, Romanowski M (2012) Light-activated content release from liposomes. Theranostics 2(10): 1020-1036

96. Li G, Mçwald H, Shchukin D (2013) Precipitation polymerization for fabrication of complex core-shell hybrid particles and hollow structures. Chem Soc Rev 42(8): 3628-3646.

97. Martínez L, Jiménez A, Hernández E (2012) Teoría de Helfrich para las membranas biológicas. Editorial Académinca Española. Saarbruken: Alemania.

98. Mittal G, Sahana D, Bhardwaj B, Kumar M (2007) Estradiol loaded PLGA nanoparticles for oral administration: effect of polymer molecular weight and copolymer composition on release behavior in vitro and in vivo. J Control Release 119(1): 77-85.

99. Nowacek A, Gendelman H (2009) NanoART, neuroAIDS and CNS drug delivery. Nanomedicine (Lond) 4(5): 557-574.

100. Silva J, Menachao F, Chorilli M (2012) Dendrimers as potential platform in nanotechnology-based drug delivery systems. IOSR J Pharm 2: 2230.

101. Thévenot J, Oliveira H, Sandre O, Lecommandoux S (2013) Magnetic responsive polymer composite materials. Chem Soc Rev 42(17): 7099 7116.

102. Unal H, d'Angelo I, Pagano E, Borelli F, Izzo A, et al. (2015) Core-shell hybrid nanocapsules for oral delivery of camptothecin: formulation development, in vitro and in vivo evaluation. J Nanopart 17: 42.

103. Woitiski CB, Neufeld RJ, Veiga F, Carvalho RA, Figueiredo IV (2010) Pharmacological effect of orally delivered insulin facilitated by multilayered stable nanoparticles. Eur J Pharm Sci 41(3-4): 556-563.

104. Woitiski CB, Sarmento B, Carvalho RA, Neufeld RJ, Veiga F (2011) Facilitated nanoscale delivery of insulin across intestinal membrane models. Int J Pharm 412(1-2): 123-131.

105. Wu ZM, Zhou LY, Guo XD, Jiang W, Ling L, et al. (2012) HP55-coated capsule containing PLGA/RS nanoparticles for oral delivery of insulin. Int J Pharm 425(1-2): 1-8.

106. Zhang ZH, Abbad S, Pan RR, Waddad AY, Hou LL, et al. (2013) N-octyl$\mathrm{N}$-arginine chitosan micelles as an oral delivery system of insulin. J Biomed Nanotechnol 9(4): 601-609. 